\title{
REVIEW
}

\section{Enhanced external counterpulsation for refractory angina pectoris}

\author{
R M Sinvhal, R M Gowda, I A Khan
}

Heart 2003;89:830-833

Enhanced external counterpulsation (EECP) is a non-invasive outpatient treatment used for angina pectoris. In patients with intractable angina refractory to aggressive surgical and medical treatment, several novel strategies are considered including EECP, transmural laser revascularisation, and spinal cord stimulation. EECP produces an acute haemodynamic effect that is presumed to be similar to that produced by the invasive intra-aortic balloon pump. By applying a series of compressive cuffs sequentially from the calves to the thigh muscles upon diastole and rapidly deflating the cuffs in early systole, an increase in diastolic and decrease in systolic pressure is created. Although data indicate improvement in angina in patients undergoing $E E C P$, the role of EECP in the treatment of angina pectoris has not yet been well defined. At present, EECP use should be limited to patients with debilitating (functional class III and IV) refractory angina pectoris who are not candidates for revascularisation, are symptomatic despite being on maximal antianginal pharmacotherapy, and have no contraindications to EECP use.

See end of article for authors' affiliations

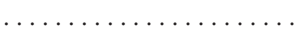

Correspondence to: Dr I A Khan, Creighton University School of Medicine, Division of Cardiology, 3006 Webster Street, Omaha, Nebraska 68131, USA;

ikhan@cardiac.creighton.edu

Accepted

24 February 2003

.......................
D espite an increasing success of conventional medical treatment and the continued development and improvement of mechanical revascularisation approaches, a significant number of patients with ischaemic heart disease and angina pectoris cannot be successfully managed, even with optimisation of medical treatment. Many of these patients are not candidates for revascularisation with angioplasty or surgery for myriad reasons. ${ }^{1}$ As the survival of patients with primary coronary events continues to increase, the number of patients presenting with coronary artery disease unsuitable to further revascularisation and symptoms refractory to medical treatment also continues to rise. Enhanced external counterpulsation (EECP) is the one of the treatment strategies that is finding a role in treatment of patients with refractory angina. $^{2}$

\section{HISTORICAL PERSPECTIVE}

Almost half a century ago Kantrowitz and Kantrowitz described diastolic augmentation as a means of improving coronary blood flow. ${ }^{3}$ Birtwell did pioneering work towards the development of the technique and showed that external compressions can be ECG gated to enhance coronary collateral circulation in experimental animal models. ${ }^{4}$ Soroff et al used the term "counterpulsation", and it initially began as an invasive internal modality. ${ }^{5}$ Since then, it has evolved as "internal" (intra-aortic balloon pump) and "external" (EECP) modes. Initially performed by cumbersome hydraulic devices, EECP has evolved into more feasible pneumatic devices. Work is ongoing towards making it transportable for use in emergency rooms and chest pain observation units. EECP has widespread geographic presence, as it has been in use in China for over two decades. Much of the Chinese experience comes from Zheng et al, who started using a sequential three cuff external counterpulsation method. ${ }^{6}$ Their positive clinical experience led to installation of thousands of EECP units in China. In 1999, the first randomised multicentre controlled trial was reported. ${ }^{7}$

\section{TECHNIQUE}

EECP involves the use of three paired inflatable cuffs wrapped around the patient's lower extremities. The cuffs are sequentially inflated during diastole, in the calves followed by lower thighs followed by upper thighs (fig 1). All pressure is released at the onset of systole. This sequential compression results in increased venous return and augmented diastolic pressure. The diastolic augmentation increases coronary perfusion pressure and provides improved afterload reduction and increased venous return with a subsequent increase in cardiac output. ${ }^{8}$ The instantaneous and simultaneous deflation of cuffs during systole also enhances systolic unloading and decreases cardiac workload by decreasing peripheral vascular resistance.

The patient is connected to an ECG monitor, as well as to a finger plethysmograph, to assess the arterial pulse augmentation. The $\mathrm{R}$ wave of the patient's ECG is used as the trigger for inflation and deflation. Pressure within the cuffs is adjustable and current EECP machines are capable of generating pressures up to $350 \mathrm{~mm} \mathrm{Hg}$, but pressures in the range of $250-275 \mathrm{~mm} \mathrm{Hg}$ are usually applied. A treatment course consists of 35 one hour sessions over a seven week period and is generally well tolerated with a low risk of adverse events.

Abbreviations: $E E C P$, enhanced external counterpulsation; IEPR, International EECP Patient Registry; MUST-EECP, multicenter study of enhanced external counterpulsation; TIMI, thrombolysis in myocardial infarction 

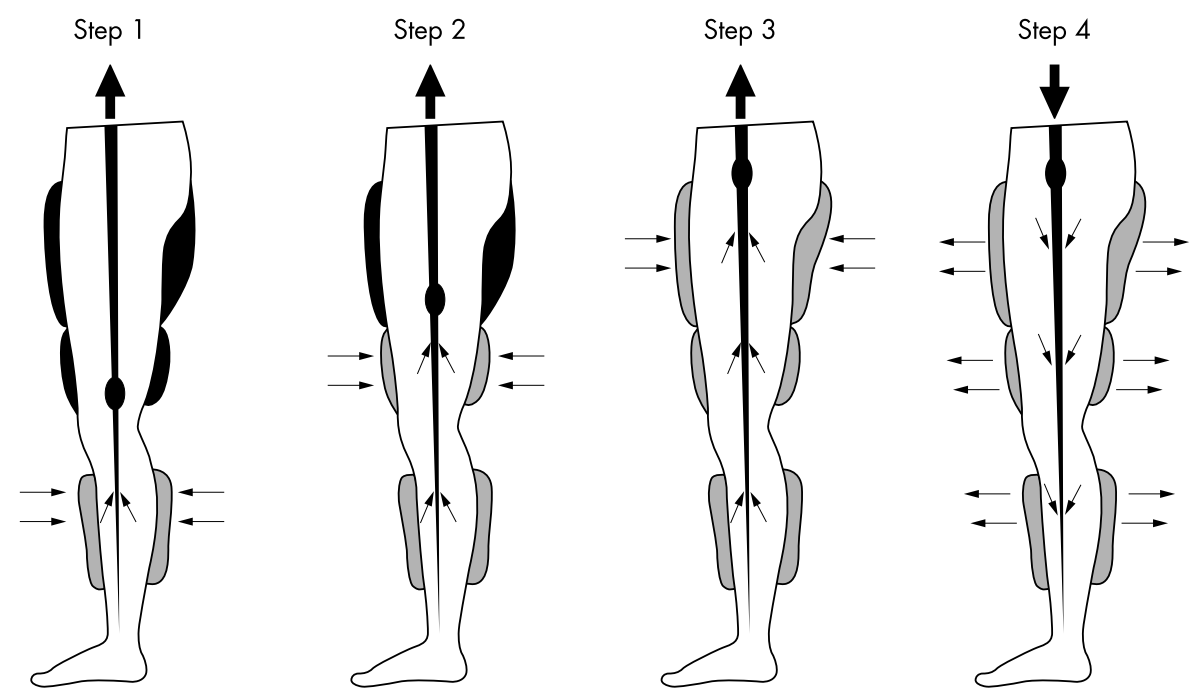

Figure 1 Technique of enhanced external counterpulsation (EECP): three pairs of pneumatic cuffs are applied to the calves, lower thighs, and upper thighs. The cuffs are inflated sequentially during diastole, calves up. The pressure is released at the onset of systole. The compression of the lower extremity venous bed increases venous return and increases diastolic pressure. Inflation and deflation are timed according to the $\mathrm{R}$ wave on the patient's cardiac monitor. The pressures applied and the inflation-deflation timing can be altered by using the pressure waveforms and ECG on the EECP monitor.

\section{MECHANISM OF ACTION}

The mechanism underlying the effects of EECP is under evaluation but several theories have been postulated..$^{8-14}$ The haemodynamic effects of EECP have been theorised to simulate the clinical use of the intra-aortic balloon pump, enhancing cardiac output, stroke volume, and retrograde aortic diastolic flow. EECP produces haemodynamic changes that reduce myocardial oxygen demand in addition to potential for increased transmyocardial pressure to open collaterals. With exposure to the augmented blood flow and endothelial shear stress, there is elaboration of nitric oxide, prostacycline, and vascular endothelial growth factor from the arterial bed that improve endothelial function and vascular remodelling. ${ }^{9}$

Lawson et al reported that EECP seemed to exert a training effect, decreasing peripheral vascular resistance and the heart rate response to exercise. ${ }^{10}$ Patients with coronary disease may improve their exercise tolerance after EECP because of both improved myocardial perfusion and a decrease in cardiac workload. Garlichs et al found reduced serum endothelin-1 concentrations (potent vasoconstrictor) by pneumatic external counterpulsation, which may explain the improved coronary perfusion and vasodilatation after EECP. ${ }^{11}$ Masuda et al showed an EECP induced increase in both resting and postexercise perfusion (coronary flow reserve representing improved collateral flow) by using ${ }^{13} \mathrm{~N}$-ammonia positron emission tomography. ${ }^{13}$ In addition, the study showed an EECP induced decrease in concentrations of brain natriuretic peptide and an increase in the concentrations of nitric oxide, suggesting an improvement in endothelial function by a neurohormonal mechanism. In another study Masuda et al examined the effect of EECP on the angiogenic factors and reported a $66 \%$ increase in human growth factor and $\beta$ fibroblast growth factor and a 33\% increase in vascular endothelial growth factor and monocyte chemoattractant protein 1 (a proinflammatory cytokine). ${ }^{14}$ Urano et al studied patients with significant coronary stenoses before and after completing 35 sessions of EECP. ${ }^{15}$ Exercise thallium, gated pool cardiac scintigraphy, left heart catheterisation, and serum assays of atrial natriuretic peptide and brain natriuretic peptide were done. All exercise parameters improved (including exercise duration and time to $1 \mathrm{~mm}$ ST depression). Exercise induced perfusion defects, left ventricular end diastolic pressure, and serum brain natriuretic peptide concentrations (which correlated with the left ventricular end diastolic pressure) were all reduced.

Michaels et al shed new light on the mechanism of action of EECP when they measured left ventricular and intracoronary haemodynamics directly among patients undergoing EECP. ${ }^{16}$
Aortic pressure, intracoronary pressure, and intracoronary Doppler flow velocity were measured at baseline and during EECP. EECP resulted in a 93\% increase in diastolic and $16 \%$ increase in mean intracoronary pressure; there was a $15 \%$ decrease in systolic pressure. Coronary blood flow, measured by Doppler and the TIMI (thrombolysis in myocardial infarction) frame count (a quantitative angiographic measure of coronary blood flow), increased by $28 \%$. The study was limited by the assessment of coronary pressures in unobstructed coronaries. Also, the attenuation of coronary flow as a function of autoregulation was not assessed, as the measurements were obtained during EECP.

In brief, it may be postulated that EECP acts by a concert of different contributing mechanisms, both cardiac and peripheral. Acute haemodynamic improvement in terms of diastolic augmentation, improved coronary perfusion and systolic unloading are supplemented by neurohormonal factors. Diastolic augmentation causes increased shear stress, stimulating endothelial growth factors promoting angiogenesis. This may help explain the long term sustained benefits of EECP even after discontinuation of treatment. EECP causes an increase in endothelial production of nitric oxide, prostacycline, and vascular endothelial growth factor and a decrease in brain natriuretic peptide.

\section{EFFICACY AND SAFETY}

Irrespective of the mechanisms postulated, there are reported benefits derived from the use of EECP with a reasonable safety profile. The efficacy of EECP was assessed in MUST-EECP (multicenter study of enhanced external counterpulsation), the first and only multicentre, prospective, randomised, blinded, placebo (sham) controlled trial on the subject. ${ }^{7}$ In this trial, conducted in seven centres, 139 outpatients with angina, documented coronary artery disease, and positive exercise treadmill test were randomly assigned to receive 35 hours of active ( $\mathrm{n}=72$ patients) or inactive $(\mathrm{n}=67$ patients) counterpulsation over a period of four to seven weeks. Fifty nine patients in the active and 65 in the inactive group completed the study. The outcome was measured in terms of exercise duration, time to $\geqslant 1 \mathrm{~mm}$ ST segment depression, average daily anginal attacks, and glyceryl trinitrate use. Patients undergoing active counterpulsation had a significant increase in time to $\geqslant 1 \mathrm{~mm}$ ST segment depression and a decrease in anginal episodes, but there was no significant improvement in the duration of exercise or glyceryl trinitrate usage. More patients in the active counterpulsation group experienced adverse events $(55 \% \vee 26 \%, \mathrm{p}<0.001)$, including device related adverse experiences (leg pain, back pain, skin 
abrasion, bruising, blistering, oedema, paraesthesia). Leg discomfort was the most frequent device related adverse effect. The non-device related adverse events were minor and generally tolerated.

The MUST-EECP was a small trial considering the prevalence and subjective nature of the anginal symptoms and, therefore, was not powerful enough to recommend EECP for routine use in patients with angina pectoris. Furthermore, the patient selection for the trial was very cautious as is evident from the long list of exclusion criteria, which further limit the use of this technique on a wide scale. The exclusions were: myocardial infarction or coronary artery bypass graft in the preceding three months; cardiac catheterisation in the preceding two weeks; unstable angina; overt congestive heart failure or left ventricular ejection fraction $<30 \%$; significant valve disease; blood pressure $>180 / 100 \mathrm{~mm} \mathrm{Hg}$; permanent pacemaker or implantable defibrillator; non-bypassed left main stenosis $>50 \%$; severe symptomatic peripheral vascular disease; varicosities; deep vein thrombosis, phlebitis or stasis ulcer; bleeding diathesis or warfarin use with international normalised ratio of prothrombin time $>2.0$; atrial fibrillation or frequent ventricular premature beats that would interfere with EECP triggering; baseline ECG abnormalities that would interfere with interpretation of exercise ECG; pregnancy or childbearing potential in women; and subjects unable to undergo treadmill testing. Another shortcoming of the MUSTEECP trial is lack of data on the rate-pressure product at the time of $1 \mathrm{~mm}$ ST segment depression before and after treatment.

Follow up analysis of patients in the MUST-EECP trial at one year showed greater improvement in the health related quality of life measures (perform activities of daily living, ability to work, bodily pain, confidence in health, energy, ability to engage in social activities with family and friends, anxiety and depression, and quality of life issues from the effects of angina on health and functioning) in the active treatment group, but it was not reported whether the decrease in anginal episode reported at the end of 4-7 weeks of active treatment with counterpulsation persisted at one year. ${ }^{17}$ Also, it was not reported whether the decrease in angina during the active treatment period was correlated with the improved health related quality of life measures at one year.

Lawson et al studied 50 patients with chronic stable angina and compared the extent of coronary disease with results of radionuclide stress testing after EECP. ${ }^{18}$ There was significant improvement in the perfusion defects after EECP, and the less the coronary disease involvement, the greater the therapeutic benefit from EECP. The presence of a patent vascular conduit, either native or bypass graft, appear to predict a favourable response to treatment. This study offers an option of palliative coronary angioplasty and coronary artery bypass grafting to decrease the disease extent and make these patients more amenable to the benefits of EECP. The benefits were sustained at five years' follow up, which showed a significant improvement in stress thallium perfusion and limiting angina. ${ }^{19}$

Suresh $e^{2} a^{20}$ performed a study examining the optimal pressures to maximise the haemodynamic benefit of EECP. EECP effectiveness ratios (ratio between diastolic augmentation and systolic unloading as measured by finger plethysmography) in the range of 1.5-2.0 were found to be optimally efficacious. ${ }^{20}$ Another study analysed the data from an EECP registry examining effect of diastolic augmentation on the efficacy of EECP. Patients who were younger, male, nonsmokers, and without multivessel coronary or non-cardiac vascular disease were likely to have higher diastolic augmentation with EECP. Patients with higher diastolic augmentation tended to have a greater reduction in angina class at six months' follow up than those with lower diastolic augmentation ratios. There is evidence that higher diastolic augmentation ratios are associated with improved short or long term clinical outcomes, suggesting that clinical benefit from EECP may be associated with the magnitude of diastolic augmentation. ${ }^{21}$

In an other report of data from an EECP consortium ( $\mathrm{n}=2289$ patients), an improvement was reported in up to $74 \%$ of patients with angina undergoing EECP by one or more Canadian Cardiovascular Society functional classes. ${ }^{22}$ The younger patients had a greater likelihood of improvement. The rate of adverse experiences was $4 \%$. The placebo effect of the device can not be ruled out, as this report is from a cohort study. In 1998, the International EECP Patient Registry (IEPR) was organised to document patient characteristics, safety, and efficacy during the treatment period, as well as long term outcomes. All centres with EECP facilities were invited to join this voluntary registry. The registry population comprises all patients starting EECP for treatment of angina pectoris in participating centres. ${ }^{23}$ The IEPR data were used to examine the benefit and safety of EECP treatment in 1957 patients, 548 with a history of heart failure. ${ }^{24}$ Angina class improved in $68 \%$ of patients. The heart failure cohort was older, with more women, a greater duration of coronary artery disease, and more prior infarcts and revascularisation procedures. Compared with patients without heart failure, significantly fewer patients with a history of heart failure completed the course of EECP and exacerbation of heart failure was more frequent in them, although the overall major adverse cardiac events (death, myocardial infarction, revascularisation) during treatment were not significantly different between the groups with and without a history of heart failure. At six months, patients with history of heart failure, although maintaining their reduction in angina, were significantly more likely to have experienced a major adverse cardiac event.

\section{CONTRAINDICATIONS}

Table 1 outlines the contraindications to EECP use.

Table 1 Contraindications to enhanced external counterpulsation (EECP)

- Within two weeks after cardiac catheterisation or arterial puncture (risk of bleeding at femoral puncture site)

- Arrhythmias that may interfere with triggering of EECP system (atrial fibrillation, flutter, and very frequent premature ventricular contractions)

- Decompensated heart failure, usually class III to IV (EECP results in an increase in venous return)

- Left ventricular ejection fraction $<30 \%$ (increased preload may precipitate heart failure)

- Moderate to severe aortic insufficiency (regurgitation would prevent diastolic augmentation)

- Severe peripheral arterial disease (reduced vascular volume and muscle mass may prevent effective counterpulsation, increased risk of thromboembolism)

- Severe hypertension $>180 / 110 \mathrm{~mm} \mathrm{Hg}$ (the augmented diastolic pressure may exceed safe limits)

- Aortic aneurysm or dissection (diastolic pressure augmentation may be deleterious)

- Pregnancy or women of childbearing age (effects of EECP on fetus have not been studied)

- Venous disease (phlebitis, varicose veins, stasis ulcers, prior or current deep vein thrombosis or pulmonary embolism)

- Severe chronic obstructive pulmonary disease (no safety data in pulmonary hypertension)

- Coagulopathy with international normalised ratio of prothrombin time $>2.0$ (to avoid risk of haematoma with high cuff pressures) 


\section{CONCLUSIONS}

Although data indicate an improvement in angina in patients undergoing EECP, the role of EECP in the treatment algorithm of angina pectoris has not yet been well defined. Large scale trials and long term data are needed to incorporate this technique into the standard treatment recommendations for angina pectoris. At present, EECP use should be limited to patients with debilitating (functional class III and IV) refractory angina who are not candidates for revascularisation, are symptomatic despite taking maximal antianginal pharmacotherapy, and have no contraindications to EECP use. In such patients, EECP, being non-invasive, may be considered before invasive techniques such as transmural laser revascularisation and spinal cord stimulation. Currently, EECP is available in highly specialised centres.

\section{Authors' affiliations}

R M Sinvhal, R M Gowda, Division of Cardiology, Beth Israel Medical Center, New York, New York, USA

I A Khan, Division of Cardiology, Creighton University School of Medicine, Omaha, Nebraska, USA

No financial support was received for this paper.

\section{REFERENCES}

1 Mannheimer C, Camici P, Chester MR, et al. The problem of chronic refractory angina: report from the ESC joint study group on the treatment of refractory angina. Eur Heart J 2002;23:355-70.

2 Kim MC, Kini A, Sharma SK. Refractory angina pectoris: mechanism and therapeutic options. J Am Coll Cardiol 2002;39:923-34

3 Kantrowitz A, Kantrowitz A. Experimental augmentation of coronary flow by retardation of arterial pulse pressure. Surgery 1953;34:678-87.

4 Clauss RH, Birtwell WC, Alberta K, et al. Assisted circulation: the arterial counterpulsator. J Thorac Cardiovasc Surg 1961;41:447-58.

5 Soroff HS, Hui J, Giron F. Historical review of the development of enhanced external counterpulsation, technology and its physiologic rationale. Cardiovasc Rev Rep 1997;18:28-32.

6 Zheng ZS, Li TM, Kambic H, et al. Sequential external counterpulsation in China. Trans Am Soc Artif Int Org 1983;29:593-603.

7 Arora RR, Chou TM, Jain D, et al. The multicenter study of enhanced external counterpulsation (MUST-EECP): effect of EECP on exercise-induced myocardial ischemia and anginal episodes. J Am Coll Cardiol 1999:33:1833-40.

8 Stys T, Lawson WE, Hui JC, et al. Acute hemodynamic effects and angina improvement with enhanced external counterpulsation. Angiology 2001;52:653-8
9 Niebauer J, Cooke JP. Cardiovascular effects of exercise: role of endothelial shear stress. J Am Coll Cardiol 1996;28:1652-60.

10 Lawson WE, Hui JC, Zheng ZS, et al. Improved exercise tolerance following enhanced external counterpulsation: cardiac or peripheral effect? Cardiology 1996;87:271-5.

11 Garlichs CD, Zhang H, Werner D. Reduction in serum endothelin-1 levels by pneumatic external compression. Can J Cardiol 1998;14:87F.

12 Taguchi I, Ogawa K, Oida A, et al. Comparison of hemodynamic effects of enhanced external counterpulsation and intra-aortic balloon pumping in patients with acute myocardial infarction. Am J Cardiol 2000;86: $1139-41$.

13 Masuda D, Nohara R, Hirai T, et al. EECP improved myocardial perfusion and coronary flow reserve in patients with chronic stable angina; evaluation by $13 \mathrm{~N}$-ammonia positron emission tomography. Eur Heart J 2001;22:16:1451-8.

14 Masuda D, Nohara R, Kataoka K, et al. Enhanced external counterpulsation promotes angiogenesis factors in patients with chronic stable angina. Circulation 2001;104(suppl II):445.

15 Urano H, Ikeda H, Ueno T, et al. Enhanced external counterpulsation improves exercise tolerance, reduces exercise-induced myocardial ischemia and improves left ventricular diastolic filling in patients with coronary artery disease. J Am Coll Cardiol 2001;37:93-9.

16 Michaels AD, Accad M, Ports TA, et al. Left ventricular systolic unloading and augmentation of intracoronary pressure and Doppler flow during enhanced external counterpulsation. Circulation 2002; 106: 1237-42.

17 Arora RR, Chou TM, Jain D, et al. Effects of enhanced external counterpulsation on health-related quality of life continue 12 months after treatment: a substudy of the multicenter study of enhanced external counterpulsation. J Investig Med 2002;50:25-32.

18 Lawson WE, Hui JC, Zheng ZS, et al. Can angiographic findings predict which coronary patients will benefit from enhanced external counterpulsation? Am J Cardiol 1996;77:1 107-9.

19 Lawson WE, Hui JC, Cohn PF. Long term prognosis of patients with angina treated with EECP: five year follow-up study. Clin Cardiol 2000;23:254-8.

20 Suresh K, Simandl S, Lawson WE, et al. Maximizing the hemodynamic benefits of EECP. Clin Cardiol 1998:21:649-53.

21 Michaels AD, Kennard ED, Kelsey SE, et al. Does higher diastolic augmentation predict clinical benefit from enhanced external counterpulsation? Data from the International EECP Patient Registry (IEPR) Clin Cardiol 2001;24:453-8.

22 Lawson WE, Hui JC, Lang G. Treatment benefit in the enhanced externa counterpulsation consortium. Cardiology 2000;94:31-5.

23 Barsness G, Feldman AM, Holmes DR, et al. International EECP Patient Registry Investigators. The International EECP Patient Registry (IEPR): design, methods, baseline characteristics, and acute results. Clin Cardiol $2001 ; 24: 435-42$.

24 Lawson WE, Kennard ED, Holubkov R, et al. Benefit and safety of enhanced external counterpulsation in treating coronary artery disease patients with a history of congestive heart failure. Cardiology $2001 ; 96: 78-84$.

\section{ELECTRONIC PAGES}

\section{eHEART: www.heartinl.com}

j

he following electronic only articles are published in conjunction with this issue of Heart.

Isolated left ventricular non-compaction: cardiomyopathy with homogeneous transmural and heterogeneous segmental perfusion

A C Borges, D Kivelitz, G Baumann

This is the first description of a case of isolated noncompaction of the left ventricular myocardium, involving a 52 year old woman presenting with progressive heart failure, with analysis of myocardial perfusion by contrast echocardiography in comparison with magnetic resonance imaging.

(Heart 2003;89:e21) www.heartjnl.com/cgi/content/full/89/ $8 / \mathrm{e} 21$
Acquired right coronary artery fistula draining to the right ventricle: angiographic documentation of first appearance following reperfusion after acute myocardial infarction, with subsequent spontaneous closure

P Schanzenbächer, J Baversachs

Most coronary artery fistulae are congenital in origin but have been reported to be acquired as complications of chest trauma, coronary angioplasty, or rupture of a coronary artery aneurysm. This is the first angiographic documentation of a coronary fistula acquired after myocardial infarction that subsequently closed spontaneously during follow up.

(Heart 2003;89:e22) www.heartjnl.com/cgi/content/full/89/ $8 / \mathrm{e} 22$ 